\section{Imaging morphogenesis of Candida albicans during infection in a live animal}

\author{
Soumya Mitra, ${ }^{a}$ Kristy Dolan, ${ }^{b}$ Thomas H. Foster, ${ }^{a}$ and \\ Melanie Wellington ${ }^{b, *}$ \\ a University of Rochester Medical Center, Department of \\ Imaging Sciences, 601 Elmwood Avenue, Box 648, \\ Rochester, New York 1462 \\ ${ }^{\mathrm{b}}$ University of Rochester Medical Center, Department of \\ Pediatrics, 601 Elmwood Avenue, Box 690, \\ Rochester, New York 14642
}

\begin{abstract}
Candida albicans is an opportunistic human fungal pathogen that requires an intact host immune response to prevent disease. Thus, studying host-pathogen interactions is critical to understanding and preventing this disease. We report a new model infection system in which ongoing $C$. albicans infections can be imaged at high spatial resolution in the ears of living mice. Intradermal inoculation into mouse ears with a $C$. albicans strain expressing green fluorescent protein results in systemic C. albicans infection that can be imaged in vivo using confocal microscopy. We observed filamentous growth of the organism in vivo as well as formation of microabscesses. This model system will allow us to gain significant new information about $C$. albicans pathogenesis through studies of host- $C$. albicans interactions in the native environment. $\odot 2010$ Society of Photo-Optical Instrumentation Engineers. [DOI: 10.1117/1.3290243]
\end{abstract}

Keywords: Candida albicans; fluorescence; confocal microscopy; in vivo imaging.

Paper 09370LRR received Aug. 21, 2009; revised manuscript received Nov. 20, 2009; accepted for publication Nov. 24, 2009; published online Jan. 13, 2010

The human fungal pathogen Candida albicans exists as a commensal organism in individuals with an intact immune system but causes opportunistic disease in patients with defects in phagocyte function or number. $C$. albicans can exist in multiple morphologies, including hyphae, pseudohyphae, and yeast forms; the ability to transition from yeast to filamentous forms (morphogenesis) is important for virulence. ${ }^{1}$ Traditional model systems for the study of $C$. albicans infections include in vitro observation of interactions between organisms and host cells and murine models of $C$. albicans disease. The most well-established animal model of $C$. albicans disease is the tail vein inoculation model. In this model, mice are injected with a large number of $C$. albicans yeast via the lateral tail vein. Outcome measurements are typically survival and kidney organism burden $48 \mathrm{~h}$ after infection, which correlates with disease severity. ${ }^{2}$

These models of $C$. albicans disease have significant limitations. In vitro studies of host-pathogen interactions are hampered by the artificial environment that lacks local environmental signals and interactions important to the function of

*Address all correspondence to: Melanie Wellington, Tel: 585-275-5944; E-mail: melanie wellington@urmc.rochester.edu leukocytes. The tail vein inoculation model relies on fairly broad outcome measures, and does not allow the researcher to study conditions in which the animal recovers from infection. The ability to image the progress of a $C$. albicans infection at high spatial resolution in vivo would enable the study of morphogenesis and host-pathogen interactions in the native host environment. Furthermore, such studies would allow investigation of the process through which an intact immune system successfully defends the host against $C$. albicans disease.

The recent expansion of in vivo imaging techniques, including confocal and multiphoton microscopy, has enabled acquisition of high resolution images of malaria ${ }^{3}$ and leishmania ${ }^{4}$ infections in intact hosts. Whole-mouse fluorescence imaging has been used to track the progression of green-fluorescent-protein (GFP)-expressing bacterial infections in internal organs. ${ }^{5}$ Bioluminescence imaging provides high contrast, low resolution images of microbial infection in vivo, and has been used for evaluation of localized C. albicans infections. ${ }^{6,7}$ Using fluorophore-conjugated antibodies to label host cells expressing major histocompatibility complex II, we recently reported confocal fluorescence images of two dendritic cell populations in the ears of living mice. ${ }^{8}$ The ability to resolve fine dendritic cell processes at depths up to $\sim 100 \mu \mathrm{m}$ suggested that we would be capable of detecting morphology in GFP-expressing C. albicans in vivo as well. One previous report describing the use of reflectance confocal microscopy to image the cornea of patients documented high contrast elongated particles in the cornea of a patient later diagnosed with $C$. albicans keratitis. ${ }^{9}$ While the particles were presumed to be $C$. albicans pseudohyphae, there was no way to definitively identify fungal elements in the clinical images. In this work, we describe the use of a new mouse model of infection and in vivo confocal fluorescence microscopy to follow $C$. albicans morphogenesis during an ongoing infection. To the best of our knowledge, this is the first experimental observation of this phenomenon in vivo and the first high resolution images of $C$. albicans infections in a living animal.

The $C$. albicans strain used in this study, YAW3, was generously provided by Drs. Konopka and Warenda (State University of New York, Stony Brook). YAW3 was constructed by integrating a construct containing yeast $\mathrm{EGFP}^{10}$ under control of the constitutively active ADH1 promoter $^{11}$ into the parental strain BWP17. Female BALB/c mice used in these experiments were maintained on chlorophyll-free chow to minimize endogenous fluorescence. Prior to injection of $C$. albicans, mice were anesthetized and hair on the ears was removed by chemical depilation. $C$. albicans yeast were then injected intradermally in a 30 - to $50-\mu$ l volume of phosphate buffered saline. As a control, the opposite ear was injected with phosphate buffered saline alone. Mice were placed on the stage in the supine position so the ventral side of the ear was facing downward for imaging. Images were acquired using a $10 \times, 0.45-\mathrm{NA}$ objective and a $100-\mu \mathrm{m}$-diam pinhole, which provides an optical section thickness of approximately $6 \mu \mathrm{m}$ as determined by fluorescence edge response measurements. ${ }^{12}$ GFP was excited at $488 \mathrm{~nm}$, and emission was detected using a 515/30 bandpass filter.

Immediately after injection, $C$. albicans yeast could be detected in the ears of living mice with signals similar to that

1083-3668/2010/15(1)/010504/3/\$25.00 @ 2010 SPIE 

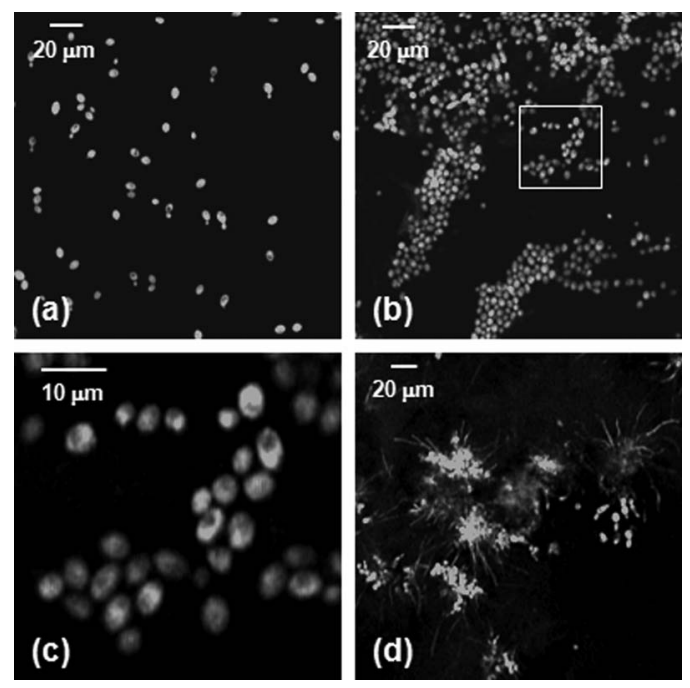

Fig. 1 Confocal images of $C$. albicans strain YAW3 in vitro and in vivo. (a) YAW3 was grown overnight, washed three times in phosphate buffered saline, placed on a microscope slide, and imaged. (b) YAW3 yeast approximately $30 \mathrm{~min}$ after an intradermal injection were imaged at a depth of $\sim 50 \mu \mathrm{m}$ in a mouse ear in vivo. (c) An expanded view of the region of interest indicated by the white box superimposed on the image in panel (b). (d) An image obtained $24 \mathrm{~h}$ after injection demonstrating in vivo morphogenesis [obtained as in panel (b)]. All images were acquired using 488-nm excitation and a $10 \times, 0.45-N A$ objective. The optical section thickness was $6 \mu \mathrm{m}$.

observed when yeast were imaged in vitro [Figs. 1(a) and 1(b)]. Yeast structures such as the vacuole can be readily detected in vivo [Fig. 1(c)]. $24 \mathrm{~h}$ after injection, organism replication resulted in the development of colony-like clusters with filamentous forms projecting out into the tissue [Fig. 1(d)]. One commonly suggested function of filamentous forms of $C$. albicans is that the filaments are responsible for invasion into new areas of tissue. While imaging alone cannot test such a hypothesis, this observed growth pattern is certainly consistent with a role for tissue invasion by filaments.

Intradermal injection of YAW3 resulted in a significant $C$. albicans infection. $24 \mathrm{~h}$ after injection, the ears injected with
$10^{5}$ or more $C$. albicans yeast/ear developed significant swelling and redness, indicating that local inflammation was occurring. In addition, the mice that received $C$. albicans injections developed ruffled fur and a hunched posture, indicating systemic illness. Mice injected with $10^{6}$ to $10^{7}$ yeast/ear developed systemic candidiasis, as detected by the presence of viable $C$. albicans in the kidneys after euthanasia. The development of systemic disease after intradermal injection of Candida demonstrates that this system successfully models dissemination of $C$. albicans infection, a critical stage in the pathogenesis of Candida.

The presence of $C$. albicans in the kidney after intradermal infection parallels the tail vein inoculation model of candidiasis, in which the kidney organism burden is monitored to follow the outcome of infection. Unlike the tail vein model, however, by using the intradermal injection we were able to use in vivo imaging to follow the progression of infection in a living animal. We observed two main patterns of infection. In the first, individual organisms were spread out throughout the tissue. In this pattern, we clearly observed the formation and growth of filamentous forms over 24 to $48 \mathrm{~h}$ (Fig. 2), with complex 3-D networks of filaments observed $48 \mathrm{~h}$ after infection. In the second pattern, we observed clusters of $C$. albicans organisms with the appearance of microabscesses [Fig. 3(a)]. These lesions increased in size over time, with some areas almost $1 \mathrm{~mm}$ in diameter $72 \mathrm{~h}$ after infection. At $72 \mathrm{~h}$, some mice were euthanized and the ears were harvested for a comparative histological evaluation. Hematoxylin-and-eosin stained slides demonstrate the extensive inflammatory infiltrate in ears injected with $C$. albicans [Fig. 3(c)] in comparison to saline injected ears [Fig. 3(b)]. Silver staining demonstrates the presence of fungal elements in the lesions [Fig. 3(d)]. Thus, both the fluorescence and histological images are consistent with microabscesses identified in the kidney after tail vein inoculation of C. albicans. $^{2}$

Using contemporary confocal fluorescence imaging techniques and a new model of invasive candidiasis, we have been able to microscopically follow the development of $C$. albicans infections in living mice. We observed morphogenesis and the formation of microabscesses, consistent with currently used models of infection. In vivo imaging is particularly relevant
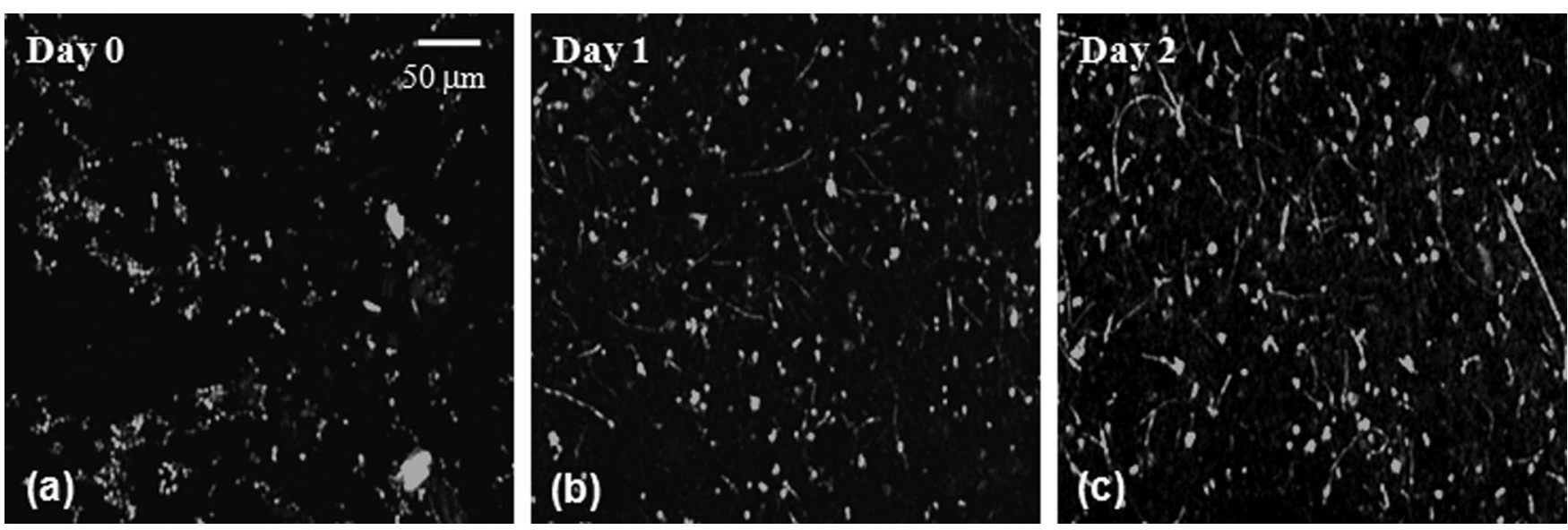

Fig. 2 Observation of $C$. albicans morphogenesis in vivo. Mouse ears were injected with C. albicans YAW3-GFP yeast on day 0 and imaged daily. Over two days, the yeast developed into a complex network of filamentous forms. Image depth and acquisition parameters were as described for Fig. 1. 

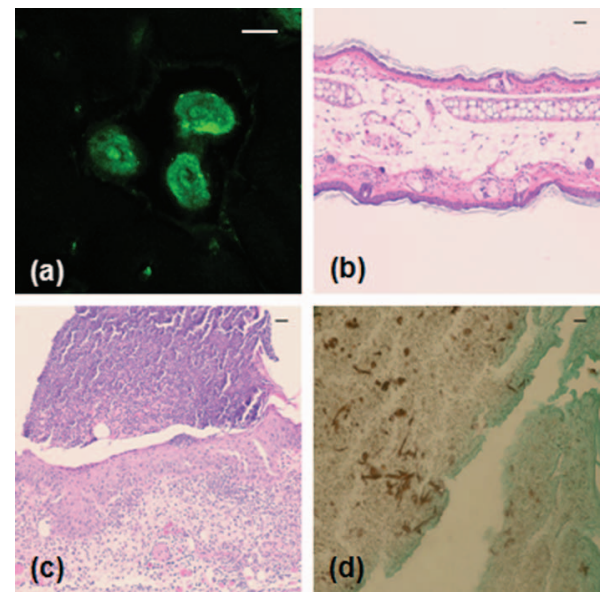

Fig. 3 Microabscess formation in infected ear tissue. $72 \mathrm{~h}$ after injection, mouse ears were imaged for fluorescence signal as in Figs. 1 and 2 , after which the mouse was then euthanized and the ears were harvested, fixed in $10 \%$ formalin, and sectioned and stained for histological analysis. (a) Representative in vivo fluorescence image illustrating the formation of microabscess. The scale bar represents $100 \mu \mathrm{m}$. Hematoxylin-and-eosin stained sections of (b) the saline injected control ear and (c) the C. albicans injected ear. (d) Sections were stained with Modified Grocott's Methenamine Silver stain (Richard Allan Scientific staining kit, VWR, West Chester, Pennsylvania) to demonstrate the presence of $C$. albicans in the tissue. Bright field images shown in (b), (c), and (d) were acquired with a 40×, 0.95-NA objective and the scale bars represent $10 \mu \mathrm{m}$.

for the study of $C$. albicans, as this opportunistic disease is dependent on host-pathogen interactions. We expect future studies using this technique will incorporate the use of multicolor imaging to analyze host-pathogen interactions, as has been performed for Leishmania. ${ }^{4}$ The use of bioluminescent C. albicans to follow infections in whole mice has been tested. ${ }^{6,7}$ However, the luciferase imaging model was designed to acquire low resolution images at the level of the entire mouse. The model system we have described has the potential to allow us to evaluate host-pathogen interactions at high spatial resolution in the intact host environment. Thus, it should significantly further our understanding of how this opportunistic fungal pathogen causes disease and responds to host defenses and antifungal therapy.

\section{Acknowledgments}

The authors are grateful to Drs. Konopka and Warenda for providing the YAW3 strain. This work was funded in part by the NIH National Center for Research Resources (ULI RR02160) and the National Cancer Institute (CA68409 and CA122093).

\section{References}

1. S. P. Saville, A. L. Lazzell, C. Monteagudo, and J. L. Lopez-Ribot, "Engineered control of cell morphology in vivo reveals distinct roles for yeast and filamentous forms of Candida albicans during infection," Eukaryot. Cell 2(5), 1053-1060 (2003).

2. D. M. MacCallum, L. Castillo, A. J. Brown, N. A. Gow, and F. C. Odds, "Early-expressed chemokines predict kidney immunopathology in experimental disseminated Candida albicans infections," PLoS ONE 4(7), e6420 (2009).

3. R. Amino, S. Thiberge, S. Blazquez, P. Baldacci, O. Renaud, S. Shorte, and R. Menard, "Imaging malaria sporozoites in the dermis of the mammalian host," Nat. Protoc. 2(7), 1705-1712 (2007).

4. N. C. Peters, J. G. Egen, N. Secundino, A. Debrabant, N. Kimblin, S. Kamhawi, P. Lawyer, M. P. Fay, R. N. Germain, and D. Sacks, "In vivo imaging reveals an essential role for neutrophils in leishmaniasis transmitted by sand flies," Science 321(5891), 970-974 (2008).

5. M. Zhao, M. Yang, E. Baranov, X. Wang, S. Penman, A. R. Moossa, and R. M. Hoffman, "Spatial-temporal imaging of bacterial infection and antibiotic response in intact animals," Proc. Natl. Acad. Sci. U.S.A. 98(17), 9814-9818 (2001).

6. T. C. Doyle, K. A. Nawotka, C. B. Kawahara, K. P. Francis, and P. R. Contag, "Visualizing fungal infections in living mice using bioluminescent pathogenic Candida albicans strains transformed with the firefly luciferase gene," Microb. Pathog 40(2), 82-90 (2006).

7. B. Enjalbert, A. Rachini, G. Vediyappan, D. Pietrella, R. Spaccapelo, A. Vecchiarelli, A. J. Brown, and C. d'Enfert, "A multifunctional, synthetic Gaussia princeps luciferase reporter for live imaging of Candida albicans infections," Infect. Immun. 77(11), 4847-4858 (2009).

8. R. J. Cummings, S. Mitra, E. M. Lord, and T. H. Foster, "Antibodylabeled fluorescence imaging of dendritic cell populations in vivo," $J$. Biomed. Opt. 13(4), 044041 (2008).

9. E. Brasnu, T. Bourcier, B. Dupas, S. Degorge, T. Rodallec, L. Laroche, V. Borderie, and C. Baudouin, "In vivo confocal microscopy in fungal keratitis," Br. J. Ophthamol. 91(5), 588-591 (2007).

10. B. P. Cormack, G. Bertram, M. Egerton, N. A. Gow, S. Falkow, and A. J. Brown, "Yeast-enhanced green fluorescent protein (yEGFP) a reporter of gene expression in Candida albicans," Microbiology 143, (Pt 2) 303-311 (1997)

11. A. J. Warenda and J. B. Konopka, "Septin function in Candida albicans morphogenesis," Mol. Biol. Cell 13(8), 2732-2746 (2002).

12. C. E. Bigelow, D. L. Conover, and T. H. Foster, "Confocal fluorescence spectroscopy and anisotropy imaging system," Opt. Lett. 28(9), 695-697 (2003). 